\title{
Ibandronat: Klinische Daten in Praxisstudie bestätigt
}

„Bereits seit 15 Jahren wird das stickstoffhaltige Bisphosphonat Ibandronat in der Therapie von Krebspatienten eingesetzt. In dieser Zeit hat Ibandronat seine Wirksamkeit und Verträglichkeit umfassend unter Beweis gestellt", konstatierte Ingo J. Diel, Mannheim. Die in klinischen Studien erhobenen Daten zu Wirksamkeit und Verträglichkeit bestätigt eine aktuelle nicht-interventionelle Studie (NIS).

Ibandronat, das zur Therapie tumorinduzierter Hyperkalzämie und zur Prävention von skelettalen Ereignissen bei Patienten mit Brustkrebs und Knochenmetastasen zugelassen ist, wird in den aktuellen Leitlinien der Arbeitsgemeinschaft Gynäkologische Onkologie erneut mit dem höchsten Empfehlungsgrad $(\mathrm{B}++)$ bewertet (ww. ago-online.de).

Phase-IIlb-Studie mit mehr als $\mathbf{3 . 5 0 0}$ Patienten

In einer aktuellen NIS reduzierte Ibandronat im Laufe des Beobachtungszeitraums von 24 Wochen bei 3.515 Patienten die anfängliche Schmerzintensität signifikant (VAS: 3,1 auf 2,3; $p<0,0001$ ). Parallel zur Abnahme der Knochenschmerzen reduzierte sich der Analgetika-Verbrauch (Patienten, die zu Studienende keine weiteren Analgetika mehr benötigten: 57\% ver-

\section{Mammakarzinom: Intra-} operative Teilbrustbestrahlung

Brusterhaltendes Operieren, insbesondere beim frühen Mammakarzinom, ist in Europa Standard. Die verbesserte Frühdiagnostik und die Etablierung neuer Strahlentherapien ermöglichen zudem stärker individualisierte, risikoadaptierte Formen der Brustkrebstherapie. So wurde vor knapp zwölf Monaten die Wirksamkeit der gezielten intraoperativen Einmalbestrahlung mit dem INTRABEAM ${ }^{\circledR}$ Gerät (Carl Zeiss Meditech) durch die TARGIT-A Studie belegt (Vaidya J et al., 2010, Lancet 376: 91-102). „Die Studie zeigt, dass bei dieser Therapieoption in der Regel über 30 Bestrahlungssitzungen für ältere Patientinnen mit kleinem Mammakarzinom ganz entfallen können bei gleicher Wirksamkeit und ebenso geringer Rückfallrate," so Jayant S. Vaidya, London/ Großbritannien. Auch nach dem Abschluss von TARGIT-A im April 2010 blieb die Rückfallrate sus 54\%). Orale und i.v. Formulierung waren vergleichbar wirksam und verträglich (Diel IJ et al., 2010, ASCO: \#1026).

\section{Pilotstudie mit Ibandronat: Direkter} Einfluss auf Tumorresterkrankung?

Eine Tumorresterkrankung wird über sogenannte disseminierte Tumorzellen im Knochenmark nachgewiesen (DTZ; Hoffmann $O$ et al., 2010, DKK: Poster 183). Die DTZ unterscheiden sich laut Studien von Tanja Fehm, Tübingen, vom Primärtumor hinsichtlich der Expression des Östrogenund des HER2-Rezeptors. Zudem handele es sich bei den DTZ um ruhende Zellen, die sich nicht teilen und proliferieren, berichtete Sabine Kasimir-Bauer, Essen. „Somit ist es zwingend notwendig, ergänzend zu den klassischen Therapien, Rezeptor-unabhängige Therapiestrategien zu finden. Bisphosphonate stehen in der Diskussion, einen Einfluss auf die Eliminierung der DTZ zu haben."

In der Pilotstudie erhielten 18 Patientinnen, die viele Jahre nach der Erstdiagnose Mammakarzinom rezidivfrei waren und bei denen DTZim Knochenmarknachgewiesen wurden, täglich 50mg lbandronat oral (Bondronat ${ }^{\circledR}$ ). Bei 15 Patientinnen konnten nach einem halben Jahr keine DTZ mehr nachgewiesen werden, bei den anderen drei Patientinnen nach weiteren sechs Monaten. „Das heißt für uns, dass das Bisphosphonat auch viele Jahre nach der Erkrankung noch Wirkung auf Tumorzellen im Knochenmark hat", kommentierte Kasimir-Bauer. Vermutlich wirke das Bisphosphonat nicht direkt auf die DTZ, sondern es inhibiere die Osteoklasten, sodass eine Änderung im sogenannten Tumor-Microenvironment entstünde, schlussfolgerte Kasimir-Bauer. FH

Quelle: Veranstaltung der Roche Pharma AG bis heute bei Patientinnen, die nur ein einziges Mal während einer brusterhaltenden Operation bestrahlt wurden, konstant niedrig. Neueste Untersuchungen zeigen nun weitere erhebliche Verbesserungen von Lebensqualität und kosmetischen Ergebnissen für TARGIT-Patientinnen nach brusterhaltender Operation. In Deutschland wird diese innovative Behandlungsmethode bereits an rund 40 zertifizierten Brustkrebszentren erfolgreich angewendet.

\section{Patienten-Ringbuch „Alles im Blick"}

Das Ringbuch „Alles im Blick“ bietet im Rahmen der von GlaxoSmithKline unterstützten Initiative "Psyche hilft Körper" eine neue Hilfestellung für Krebspatienten: Es unterstützt die Patienten dabei, ihren Behandlungsverlauf zu dokumentieren und zu organisieren. Zusätzlich enthält das Ringbuch Tipps zu Behandlungsformen sowie ein Register mit medizini- schen Fachausdrücken. Auch die aktuelle Ausgabe des Adressverzeichnisses „Sprechstunde für die Seele", in der bundesweit Psychoonkologen aufgeführt sind, liegt dem Ringbuch bei. Das Ringbuch kann kostenfrei über den behandelnden Arzt bezogen werden. Weitere Informationen zur Initiative „Psyche hilft Körper “ gibt es auf www.gsk-onkologie.de unter dem Punkt Informationsangebote für Patienten.

\section{Neuausbietung Oxaliplatin}

Medoxa ${ }^{\circledast}$ (Oxaliplatin) als Konzentrat zur Herstellung einer Infusionslösung wurde von der medac GmbH in Deutschland im Februar 2011 neu eingeführt. Das Präparat wird in einer Konzentration von $5 \mathrm{mg} / \mathrm{ml}$ in den Wirkstärken $50 \mathrm{mg}, 100 \mathrm{mg}$ und $200 \mathrm{mg}$ angeboten. Medoxa ${ }^{\varpi}$ ist in Kombination mit 5-Fluorouracil und Folinsäure zugelassen für die adjuvante und die palliative Therapie des kolorektalen Karzinoms. 